\title{
Thermal signature of the buried mine - model and experiment
}

\author{
by P. Pregowski ${ }^{1}$, W. Swiderski ${ }^{2}$, R.T. Walczak ${ }^{3}$, K. Lamorski $^{3}$ \\ ${ }^{1}$ PIRS, Pregowski Infrared Services, st. Zachodzacego Slonca 36, 01-495 Warsaw, Poland \\ 2 Military Institute of Armament Technology (MIAT), st. Prymasa Wyszynskiego 7, Zielonka, Poland \\ ${ }^{3}$ Institute of Agrophysics, Polish Academy of Sciences, st. Doswiadczalna 4, 20-236 Lublin, Poland
}

\begin{abstract}
The main disadvantage of applying IR thermal images for detection of buried mines, is the presence of various false indications in thermograms. Selected results of research works for using infrared thermography for buried mines detection are presented in the paper. Developed numerical model describing heat and water transfer phenomenon in the soil has been verified by laboratory experiment. The aim of presented models was to help in recognising the peculiarities of signal and noises depending on such parameters as: time and space variability of moisture and density of soil, buried mine and soil features and environmental conditions.
\end{abstract}

\section{Introduction}

In recent years, many world-wide institutions have started the work to improve mine disposal effectiveness. Among about 20 technologies presently developed, IR imaging is one of essential ones - although strong dependence of results on both measuring conditions and operator skills is a weakness of this method. To describe mine's image in the output of thermal imager, a few different models have to be combined. Models to describe the influence of the IR camera features and models to predict the radiometric aspects of incoming signals, almost similar for land and buried mines, appeared to be relatively well developed. Study of the physics-related papers ${ }^{1-5}$ described thermal detection of buried mines, showed this problem as not developed enough.

Many factors which depend on mine placing environment, existing atmospheric conditions and thermal characteristics of mine itself play their role in creation of buried mine thermal image on the ground surface. Very high level of variation is typical for atmospheric conditions and this is what makes great difficulties in determination of particular parameters influence on mine detection. Computer simulation of the phenomenon as well as verifying laboratory tests of sand buried mine were carried out in order to remove interference caused by variable environment conditions and to get more detailed knowledge of phenomena physics providing thermal image of buried mine.

\section{Modelling}

\subsection{Signals incoming to IR camera}

Underground objects of passive type, can manifest themselves only in result of changes in heating/cooling conditions. IR camera detects energy, radiated from investigated surface, and from the atmosphere. It causes specific needs to consider the detection of a buried mine as environment and time-dependent processes. The basic equations and discussion of different aspects of these problems modelling had been presented earlier ${ }^{6}$.

\subsection{Determination of the soil thermal properties}

The main problem in studying thermal relations in the soil is getting to know and analysing the reasons that influence the final heat effect in the soil, i.e. its temperature. The accuracy of soil temperature estimations depend both on the model applied, initial and boundary 
conditions, and tangibility of thermal properties in the studied profile, as well as on accuracy of determinations. Determination of soil thermal properties, mainly the thermal conductivity of soil, present the most difficult problem ${ }^{7}$. Thermal conductivity of the soil has been estimated by means of statistical-physical model elaborated in IA PAS Lublin ${ }^{8}$. It takes mineral fractions of soil, soil density, water content and other soil parameters into consideration. In this model, the volumetric unit of soil is treated as a system, made of elementary geometric spheres which form overlapping layers. The average value of thermal conductivity of the soil, is estimated from resulting thermal resistance and average resistance of the corresponding flat layer. The soil volumetric heat capacity has been calculated from the de Vries formula ${ }^{9}$. Both of these parameters allow to calculate thermal diffusivity of the soil .

The application of mentioned models allows to determine all basic soil thermal characteristics in dependence of soil humidity $\left(\Theta_{v}\right)$, soil density $(\rho)$, soil temperature $(T)$, mineral soil composition $\left(f_{i}\right)$.

\subsection{Water and heat transfer model in soil}

Temperature distributions on the soil surface strongly depend on the state of the processes of mass and energy exchange (radiation and convection, evaporation and water condensation, supply of water through precipitation and gaseous exchange). It was assumed that soil medium is homogeneous and parameters describing this medium are changeless in the whole its volume except that they depends on soil temperature and humidity.

Following processes were taken into account at physical description of the problem:

- water conductivity resulting from soil water potential gradients,

- thermal conductivity resulting from temperature gradients,

- surface soil processes: vaporising and energy exchanging with the environment.

Conductivity of water existing in the form of vapour and heat conductivity caused by possible absorption and emission of energy at water vaporising and condensation of vapour in the soil were not taken into account.

Mine is to be considered as a roller (Fig.1)., so all the system, soil and buried mine, has axial symmetry.

Left part of the figure shows real geometry of the simulated problem. Right part shows geometry to which this problem reduces, while we assume axial symmetry - so there would be no flow through internal boundary (symmetry axis).

\section{Boundary conditions:}

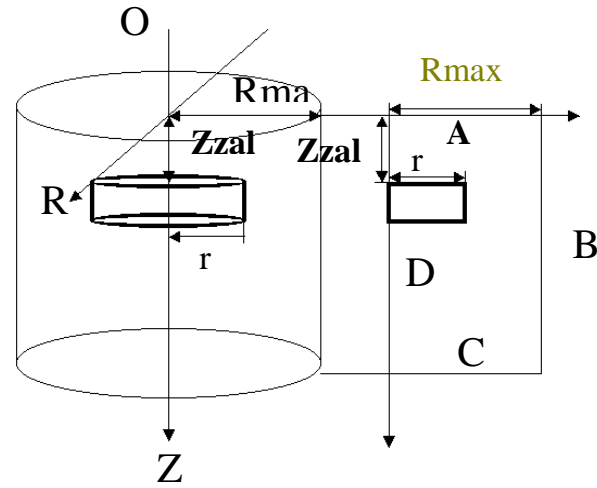

Fig.1

A - heat and water fluxes depend on the atmospheric conditions:

$$
R_{n}+H_{S}+L E+G=0
$$

where: $\mathrm{Rn}$ - net radiation at the soil surface, $\mathrm{Hs}$ - sensible heat flux exchanged between soil surface and atmosphere, $L$ - latent heat of vaporising, $E$ - water flux at the soil surface, G - the heat flux at the soil surface;

$B$ - no flow through this boundary;

C - no flow or constant value of temperature or (and) water content on this boundary;

D - no flow through this boundary, this is axis of symmetry. 


\section{Basic physical model equations:}

Transport of water in the soil profile (Richards equation): $C_{w}(h) \frac{\partial h}{\partial t}=\nabla(K(h) \nabla(h+z))$

where: $C_{w}(h) \equiv \frac{\partial \theta}{\partial h}$ - soil water capacity $\left[\frac{1}{m}\right], K(h)$-unsaturated soil hydraulic conductivity $\left[\frac{m}{s}\right]$ $h$ - pressure head (soil water potential) $\left[\mathrm{mH}_{2} \mathrm{O}\right], z$-distance from reference level $[\mathrm{m}]$, $\theta$ - volumetric soil water content $\left[\frac{\mathrm{m}^{3}}{\mathrm{~m}^{3}}\right]$

Heat transport in the soil profile: $C_{v}(\theta) \frac{\partial T}{\partial t}=\nabla(\lambda(\theta) \nabla T)$

where: $C_{v}(T, \theta)$ - volumetric soil heat capacity $\left[\frac{J}{m^{3}{ }^{o}}\right], \lambda(\theta)$ - soil heat conductivity $\left[\frac{W}{m^{o} C}\right]$, $T$ - soil temperature $\left[{ }^{o} \mathrm{C}\right]$

\section{Supporting formulas:}

Soil water parameters are evaluated by Mualem and van Genuchten model:

* the soil water content (retention curve): $\theta=\theta_{R}+\left(\theta_{S}-\theta_{R}\right)\left[\frac{1}{1+(\alpha h)^{n}}\right]^{1-1 / n}$

where: $\theta_{R}, \theta_{S}$ - residual and saturated water content, $\alpha, n$ - empirical parameters

* the soil water capacity function: $C_{w}(h) \equiv \frac{\partial \theta}{\partial h}$

* the soil hydraulic conductivity: $K(h)=K_{S} \frac{\left\{1-(\alpha h)^{n+1}\left[1+(\alpha h)^{n}\right]^{\frac{1-n}{n}}\right\}^{2}}{\left[1+(\alpha h)^{n}\right]^{\frac{n-1}{2 n}}}$

where: $K_{S}$ - is saturated hydraulic conductivity, a parameter which has to be measured

* The net radiation at the soil surface: $R_{n}=(1-a l)(1-\omega) I_{G}+\omega I_{G}+R 1-\varepsilon \sigma(T+273.16)^{4}$ where: al - albedo, $I_{G}$ - global radiation, $\varepsilon$ - soil surface emissivity, $\sigma$ - St.-Boltzmann constant, R1 - long-wave radiation of atmosphere, $\omega$ - infrared to visible light ratio in global radiation.

* The long-wave radiation of atmosphere: $\quad R 1=\sigma\left(T_{a}+273.16\right)^{4}\left[0.605+0.048\left(1370 H_{a}\right)^{1.2}\right]$ where $T_{a}$ is temperature of air $\left[{ }^{\circ} \mathrm{C}\right]$ and $H_{a}$ is absolute air humidity $\left[\mathrm{kg} / \mathrm{m}^{3}\right]$.

* Sensible heat flux $H_{S}$ : $H_{S}=\rho_{a} c_{p a}\left(T_{S}-T_{a}\right) / r_{a}$

where: $\rho_{a}$ is air density $\left[\mathrm{kg} / \mathrm{m}^{3}\right], c_{p a}$ is specific heat of air at constant pressure $\left[\mathrm{J} / \mathrm{kg}^{\circ} \mathrm{C}\right]$

$r_{a}$ is the aerodynamic boundary layer resistance $[\mathrm{s} / \mathrm{m}]: \quad r_{a}=\frac{\ln \left(2 / z_{o}\right)^{2}}{0.16 W_{s}}$

(10)

where $z_{o}$ is roughness length $[\mathrm{m}]$, and $W_{s}$ is the wind speed $[\mathrm{m} / \mathrm{s}]$.

* Flux of the water evaporating from the soil surface: $E=\left(H_{o}-H_{a}\right) / 1000 r_{a}$

where $H_{0}$ is air humidity near the soil surface and $H_{a}$ is the air humidity above the soil.

Physical model was numerically implemented by using finite difference method on rectangular net. As a result, the system of two partial differential equations was approximated 
by system of few algebraic equations. Iterative methods were used to find solutions of this equation's system in consecutive time steps.

\section{Experimental setup}

Matching of the measurement conditions accordingly with model assumptions was the most important task at preparing of laboratory measurement set-up (Fig.2).

An isolated chamber with controlled temperature and humidity was used to build this set-up.

The main performances of such laboratory-testing centre are:

- lack of surface interference's (lack of wind, fallout and flora influence),

- constant and predictable base ground humidity conditions,

- homogeneous granulometric conditions,

- settled thermal conditions for measurement instrumentation,

- accurate field parameters control (heating, wetting, hardening level).

Fig. 2

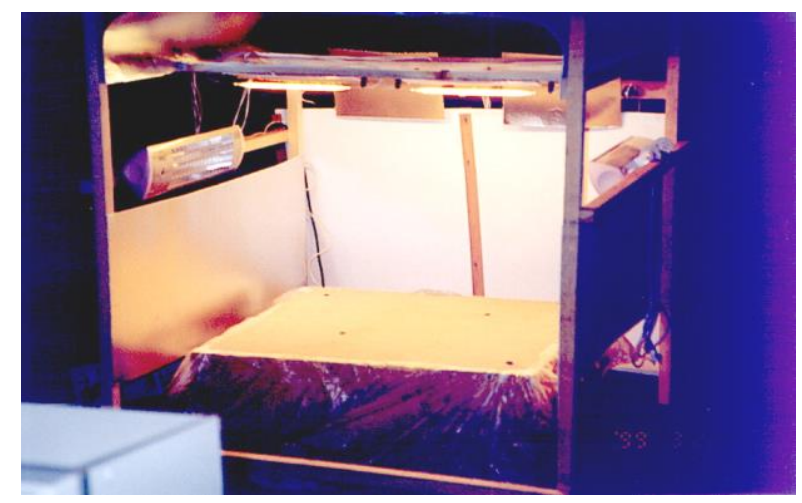

Measurement field takes the central part of the set-up and wooden container with dimension $900 \times 750 \times 300$ and isolated by foil is its main component. The temperature sensors and mine were placed inside of the sand filled container in precisely determined spots. Inside edge dimensions of the container are determined by installed lamps. Granulometric structure of the sand used for filling of the container was estimated. The experiments were based on the observation of the temperature changes during heating and cooling the surface soil using electrical lamps which delivered a uniform illumination up to $600 \mathrm{~W} / \mathrm{m}^{2}$. Metallic or plastic mine was placed in the middle of the box at depth $5 \mathrm{~cm}$. All investigations were carried out using the AGEMA 900 SW and LW thermal cameras supported by set of other meters to control measuring conditions inside and outside the box.

Thermodynamic balance of the measured object was the main requirement before starting the test. And for that reason all disturbances (displacement of the mine, refillment of the sand losses, sensor position correction etc.) were carried out a few days before planned measurement session.

\section{Results}

Described models allowed us to determine the basic thermal characteristics of the soil with relation to water content $(\theta)$, soil density $(\rho)$ and temperature $\left(T_{s}\right)$.

Fig. 3-4 concern the most popular soil i.e. sand and show influence of wetting as well as soil compacting $(\rho)$ for its thermal conductivity and diffusivity. These relationships are non-linear

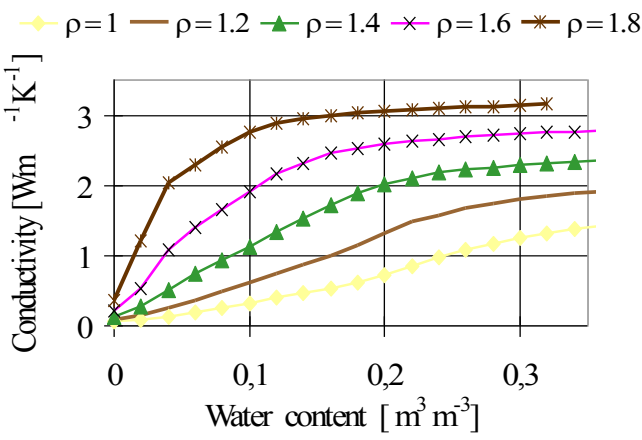

Fig.3 Thermal conductivity of sand

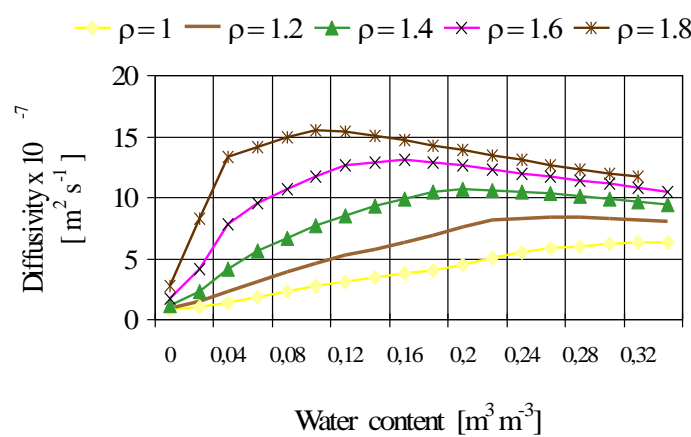

Fig.4 Thermal diffusivity of sand 
in character. It is important to observe that maxim of the thermal diffusivity occur at lower moisture contents for higher densities and that with an increase in the soil density the maxim of the thermal diffusivity tend towards lower moisture content values. For the soils with low moisture even low changes of soil density cause very big changes of the thermal features of this medium.

Fig. 5 shows that influence of the soil temperature changes on thermal diffusivity is much lower than moisture. Similar relationships for the case of the thermal conductivity dependence from temperature changes were observed.

Fig.5

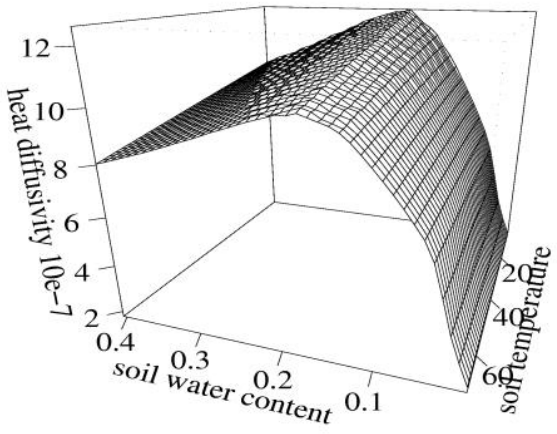

Fig. 6 presents thermal visibility of the mines buried at depth of $5 \mathrm{~cm}$, - resulted from various amplitudes of the solar irradiation(200-500) $\mathrm{Wm}^{-2}$ and unchanged cyclic changes the air temperature and the solar heating versus time. Inversions of thermal signatures and their delay in time, caused by both differences between solar activity and air temperature maximums as well as top layer thickness influence, are clearly seen. Fig. 7 shows simulated temperature distributions in soil at the end of heating (a) and after long cooling (b) the sand with buried metallic mine. Fig. 8 is simulation of the thermal signature for the same mine at the end of heating.

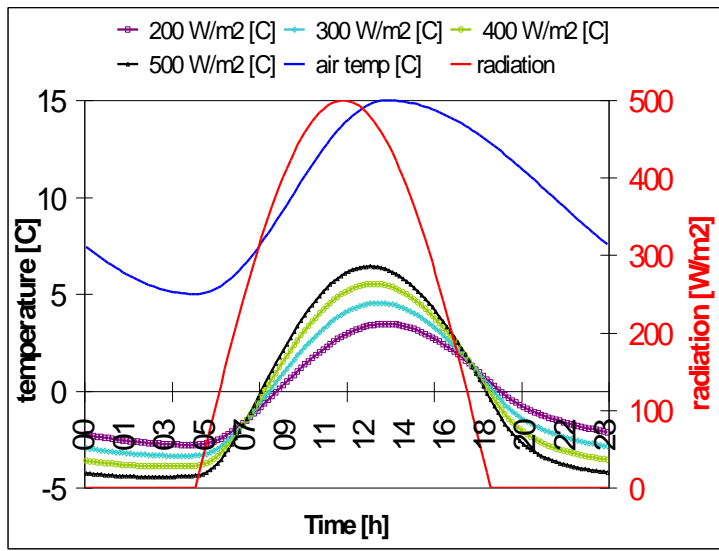

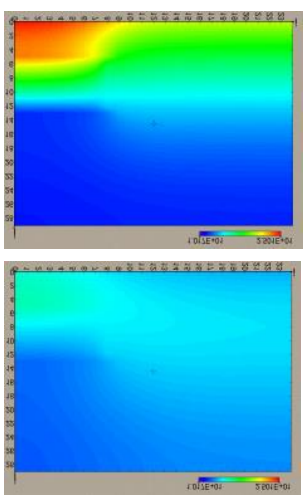

Fig. 6 Fig. $7 \mathrm{a}, \mathrm{b}$

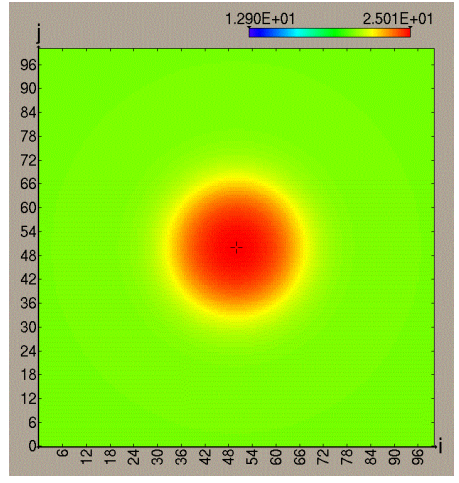

Fig. 8

Fig. 9 presents typical example of IR thermograms of plastic mine for different time of heating and cooling processes recorded at above described laboratory stand.

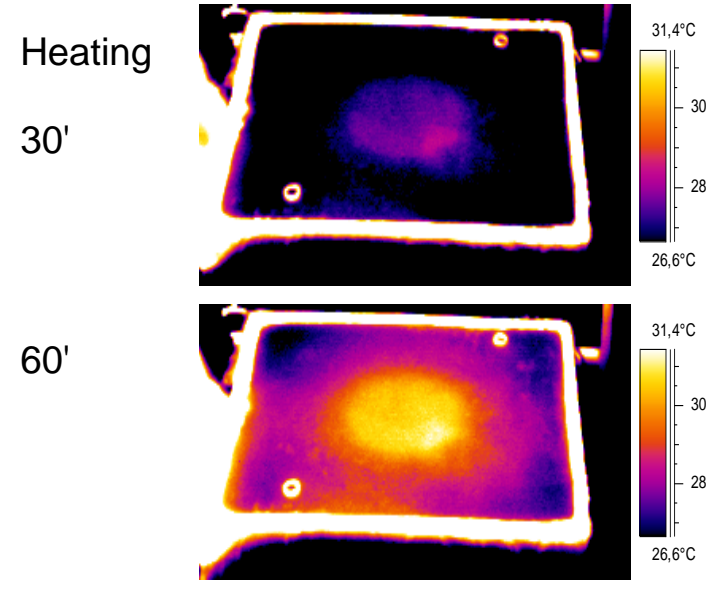

Cooling

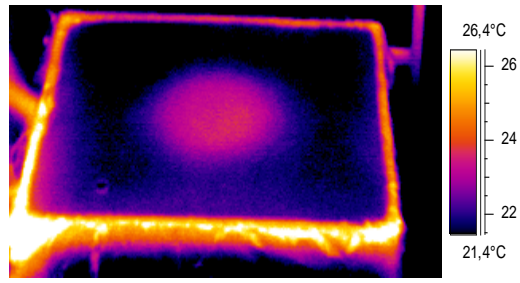

$120^{\prime}$

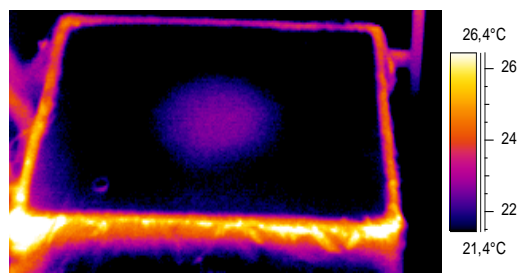




\section{Conclusions}

The elaborated model enabled us to use simulation prediction as the basis for experiments, which otherwise would be difficult or impossible to perform. Correctness of assumptions for developed physical-mathematical model of water flow in soil has bee confirmed by laboratory experiment. Obtained results in quantitative form, explain some causes of troubles in determining what is what and why just the particular spot marks the mine - proving a few weak sides of the thermographic method: even weak differences in soil moisture and/or density distributions can act as sources of false alarms. These relationships occur particularly strong for low moisture and high density of soil. The importance of these effects decreases when diurnal amplitude of temperature changes increases. To increase the model's accuracy in representation of the real-world, some modernisation, as e.g. more detailed description of the surface feature influences and conductivity of water vaporizing or energy changes caused by water vaporising and condensation of vapour in the soil are planned. In the present state, elaborated models are unsuitable for the field application. Similar tests in the field, although much more complicated and time consuming, are planned for this summer and autumn and will be executed on the specialised agro-climatical stand in IA PAS Lublin.

\section{Acknowledgement}

We would like to recognise help provided by B. Usowicz from Institute of Agrophysics and D. Szabra from MIAT. Research was supported by the State Committee for Scientific Research under biennial project No 0-T00A-015-14, finished at 1999.

\section{REFERENCES}

[1] MAKSYMONKO (G.), WARE (B.), POOLE (D.) - A characterisation of diurnal and environmental effects on mines and factors influencing the performance of mine detecting ATR algorithms, Proc. of Conf. Detection Technologies for Mines and Minelike Targets, SPIE, Vol.2496, 1995, p.140-151.

[2] SIMARD (J-R.)- Improved Landmine Detection Capability (ILDC): Systematic approach to the detection of buried mines using passive IR imaging, Proc. of Conf. Detection Technologies for Mines and Minelike Targets, SPIE, Vol.2765, 1996, p.489-500.

[3] JANSSEN (Y.H.L.), DE JONG (A.N.), WINKEL (H.), VAN PUTTEN (F.J.M.) - Detection of surface laid and buried mines with $I R$ and $C C D$ cameras, an evaluation based on measurements, Proc. of Conf. Detection Technologies for Mines and Minelike Targets, SPIE, Vol.2765, 1996, p.448-459.

[4] RUSSEL (K.), MCFEE (J.), SIROVYAK (W.) - Remote performance prediction for IR imaging of buried mines, Proc. of Conf. Detection Technologies for Mines and Minelike Targets, SPIE, Vol.3079, 1997, p.762-769.

[5] STORM (J.), HAUGSTED (B.) -Detection of buried mines facilitated by actively provoked IR signature, Proc. of Conf. Detection Technologies for Mines and Minelike Targets, SPIE, Vol.3710, 1999, p.167-172.

[6] PREGOWSKI (P.), SWIDERSKI (W.), WALCZAK (R.T.), LAMORSKI (K.) - Buried mine and soil temperature prediction by numerical model, Proc. of Conf. Detection Technologies for Mines and Minelike Targets, SPIE AeroSense Conf., Orlando, 25-27 April, 2000 (in the process).

[7] WALCZAK (R.T.), USOWICZ (B.) - Soil temperature prediction by numerical model, Polish Journal of Soil Science, 1995, p.86-94.

[8] USOWICZ (B.) - Statistical-Physical Model of Thermal Conductivity in Soil., Pol. J. Soil. Sci., 25, 1992, p. 25-34. 